\title{
Bifurcation, chaotic and hysteresis phenomena of broadband tristable energy harvesters
}

\author{
Shengxi Zhou ${ }^{1}$, Junyi Cao², and Grzegorz Litak ${ }^{3,4, a}$ \\ ${ }^{1}$ School of Aeronautics, Northwestern Polytechnical University, Xi'an, 710072, China \\ ${ }^{2}$ School of Mechanical Engineering, Xi'an Jiaotong University, Xi'an, 710049, China \\ ${ }^{3}$ Lublin University of Technology, Faculty of Mechanical Engineering, Nadbystrzycka 36, PL-20-618 Lublin, Poland \\ ${ }^{4}$ Department of Process Control, AGH University Science and Technology, Mickiewicza 30, PL-30-059 Krakow, Poland
}

\begin{abstract}
We analyze energy harvesting using a mechanical resonator with three potential wells. Nonlinear effects are leading to frequency broadband voltage response via bifurcations, multiple solutions and dynamical hysteresis. We show the characteristic features of the obtained solutions. In particular, basins of attraction of solutions are discussed.
\end{abstract}

\section{Introduction}

Vibration energy harvesting has been expected to be a promising way to solve the challenging issue of the permanent energy supply for small sensors and MEMS devices [1-5]. By far, the response characteristics and energy harvesting performance of nonlinear monostable and bistable energy harvesters have been widely revealed and verified [6-13]. Among currently existing energy harvesters, the tristable energy harvester (TEH) is one of most recent energy harvesters [14-17]. More importantly, the TEH was theoretically and experimentally verified that it has very good energy harvesting performance under low-frequency and low-level excitations. However, there is still missing deep investigation on the dynamic response of the TEH. Therefore, this paper explores the bifurcation, chaotic and hysteresis phenomena of broadband TEHs based on simulation and experiments. In detail, the bifurcation phenomena of the TEH are analyzed by using Poincaré maps and the phase portrait topology. The responses of the TEH under linearly increasing frequency sweep excitation and decreasing frequency sweep excitation clearly demonstrate its existing dynamical hysteresis phenomena (Fig. 1).

The schematic diagram of the TEH is shown in Fig. 1. The harvester mainly includes a piezoelectric beam, several magnets, a load resistance and a supporting mechanism. The nonlinear magnetic force can be produced by the interaction among endmost magnets and external magnets, which depends on the relative position of these magnets. Therefore, the equivalent nonlinear restoring force is the vector sum of the linear restoring force of the piezoelectric beam and the nonlinear magnetic force. The TEH has five equilibrium positions (1, 3 and 5 are stable equilibrium positions, while 2 and 4 are unstable equilibrium positions) [14-17]. Under the base displacement excita-

ae-mail: g.litak@pollub.pl

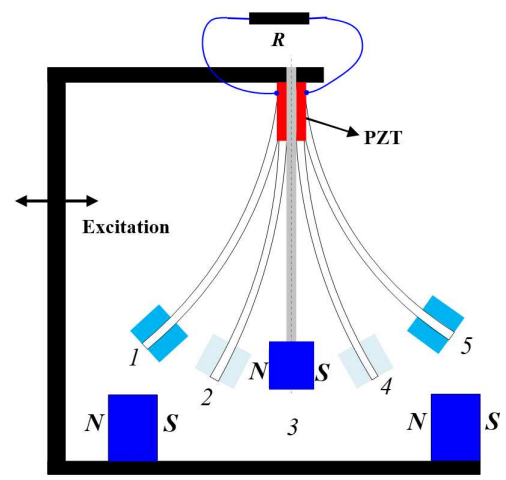

Figure 1. Schematic diagram of the tristable energy harvester.

tion, the governing model of the THE can be described by the following equations [16]:

$$
\begin{aligned}
& m \ddot{y}(t)+c \dot{y}(t)-F_{r}(y)-\theta v(t)=F(t), \\
& C_{p} \dot{v}(t)+v(t) / R+\theta \dot{y}(t)=0,
\end{aligned}
$$

where $m$ and $c$ are the equivalent mass and the equivalent damping, respectively; $y(t)$ is the tip horizontal displacement relative to the base dependent on time; $C_{p}, R$ and $\theta$ are the equivalent capacitance, the load resistance and the equivalent electromechanical coupling coefficient, respectively; $V(t)$ is the output voltage (it is also the voltage at the load resistance $R) ; F_{r}$ in the equivalent nonlinear restoring force, with accuracy to the sign, while $F(t)$ is the modal excitation force.

This paper mainly focuses on investigating the general dynamic response characteristics of the TEH for enhancing vibration energy harvesting performance. Therefore, a dimensionless model of the TEH is studied. Under a 


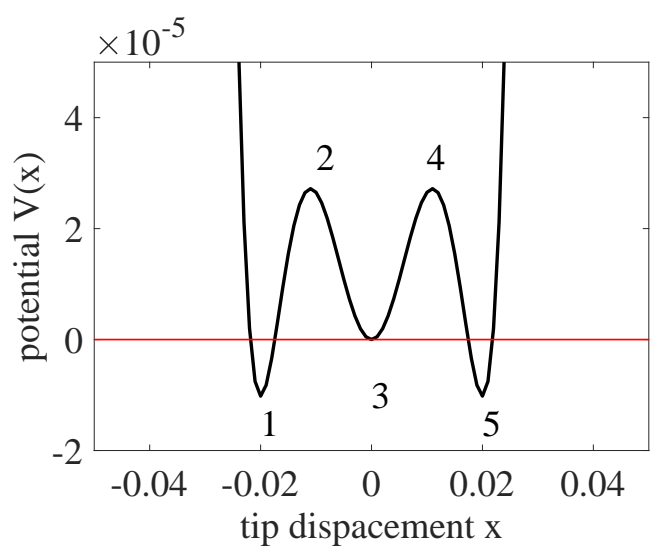

Figure 2. Potential of the examined TEH system with a tristable characteristic. Numbers $1-5$ correspond to stable $(1,3,5)$ and unstable $(2,4)$ equilibria, respectively.

harmonic base acceleration excitation $A \cos (\omega t)$, the dimensionless dynamic model of the TEH can be described by the following equations [16]:

$$
\begin{aligned}
& \ddot{x}(t)+\xi \dot{x}(t)-F_{r}(x)-\theta v(t)=A \cos (\omega t), \\
& C_{p} \dot{v}(t)+v(t) / R+\theta \dot{x}(t)=0,
\end{aligned}
$$

where $\xi$ is the dimensionless damping coefficient. $F_{r}(x)$, dependent on the dimensionless displacement $x$ can be expressed as:

$$
F_{r}=-\left(x-\mu x^{3}+\beta x^{5}\right)
$$

where $\mu$ and $\beta$ are the coefficients of the cubic $x^{3}$ and quintic $x^{5}$ terms of the equivalent nonlinear restoring force, respectively; the coefficient of $x$ is set to be one for analysis. In order to make five roots (to yield a tristable characteristic), $F_{r}$ should meet a specific condition which can be deduced based on Eq. (5). Then, the potential energy function can be expressed as (see also in Fig. 2):

$$
V(x)=\frac{1}{2} x^{2}-\frac{1}{4} \mu x^{4}+\frac{1}{6} \beta x^{6} .
$$

It is noted that all the parameters are dimensionless for numerical analysis, while these parameters can be theoretically calculated or experimentally measured based on a specific energy harvesting device. In this study, the dimensionless system parameters are set to: $\xi=0.8, C_{p}=0.05$, $\theta=0.5, R=1.0 \times 10^{7}, \mu=-1.0764 \times 10^{4}, \beta=2.0661 \times 10^{7}$.

\section{Basins of attraction of the competing solutions}

Basins of attraction of TEH are explored below. In this paper, coexisting orbits are named as interwell attractor and intrawell attractor, and their basins of attraction are depicted in white and black colors, respectively. Chaotic, quasi-periodic, or harmonic motions were not specifically distinguished. Basins of attraction were obtained based on the numerical calculation from these initial conditions via

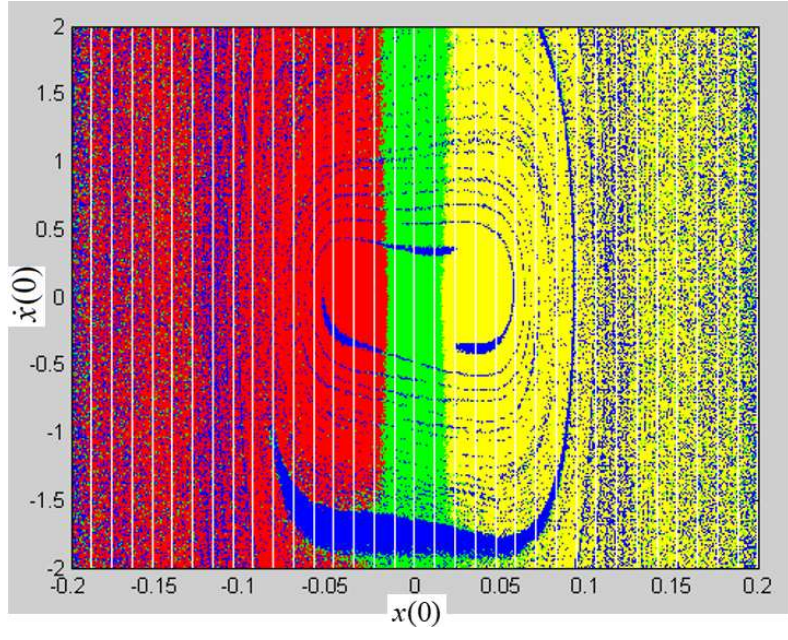

Figure 3. Basins of attraction: the excitation frequency $f=8 \pi$ and the excitation amplitude $A=1.2$. Red, green and yellow colours correspond to basins of intrawell oscillatory solutions around equlibria 1,3,5, respectively, while blue correspond to basin of interwell oscillations.

MATLAB software. Initial velocity and initial displacement were varied from -2.0 to 2.0 and -0.2 to 0.2 , respectively. The interwell oscillation phase, and the intrawell oscillation phases around equilibrium point 1, 3 and 5 are depicted in blue, red, green and yellow colors, respectively. In Fig. 3, the excitation frequency $\omega=8 \pi$ and the excitation amplitude $A=1.2$ were selected. It was found that $20.8 \%$ of initial conditions yield high-energy interwell oscillations. The proportion of initial conditions yielding intrawell oscillations equilibrium point 1,3 and 5 is $33.8 \%, 11.2 \%$ and $34.2 \%$, respectively. Keeping $A=1.2$ and increasing $\omega$ to $9 \pi$ the proportion of initial conditions which yield high-energy interwell oscillations decreased to $4.8 \%$, as shown in Fig. 3. Meanwhile, the proportion of initial conditions yielding intrawell oscillations equilibrium point 1,3 and 5 is increased to $41.1 \%, 13.1 \%$ and $41.0 \%$ respectively. When $\omega$ is further increase to $10 \pi$ (with the same $A=1.2$ ), only $2.1 \%$ initial conditions yield high-energy interwell oscillations, as shown in Fig. 4. The proportion of initial conditions yielding intrawell oscillations equilibrium point 1 and 5 becomes exactly the same and is $42.9 \%$. This tendency indicates that the TEH can be much easier to achieve high-energy interwell oscillations under a low excitation frequency with the same excitation amplitude. It is also worth to notice that the solutions are depicted with different colours (red, green, yellow and blue). The basins demonstrate fractal borders indicating existence of the chaotic solutions. Fractal mixing of three basins of attraction indicate the Wada patterns [18, 19].

\section{Experimental results: dynamical hysteresis}

For the assumed system (Fig. 1) we performed experimental studies. As analyzed above, the TEH has numerical multi-solution ranges and hysteresis phenomenon 


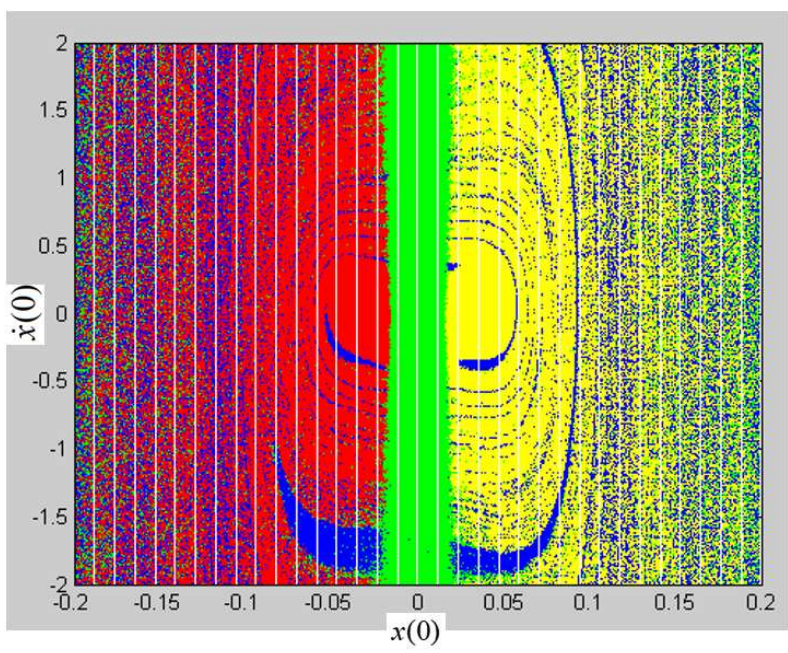

Figure 4. Basins of attraction: the excitation frequency $f=9 \pi$ and the excitation amplitude $A=1.2$. Red, green and yellow colours correspond to basins of intrawell oscillatory solutions around equlibria $1,3,5$, respectively, while blue correspond to basin of interwell oscillations.

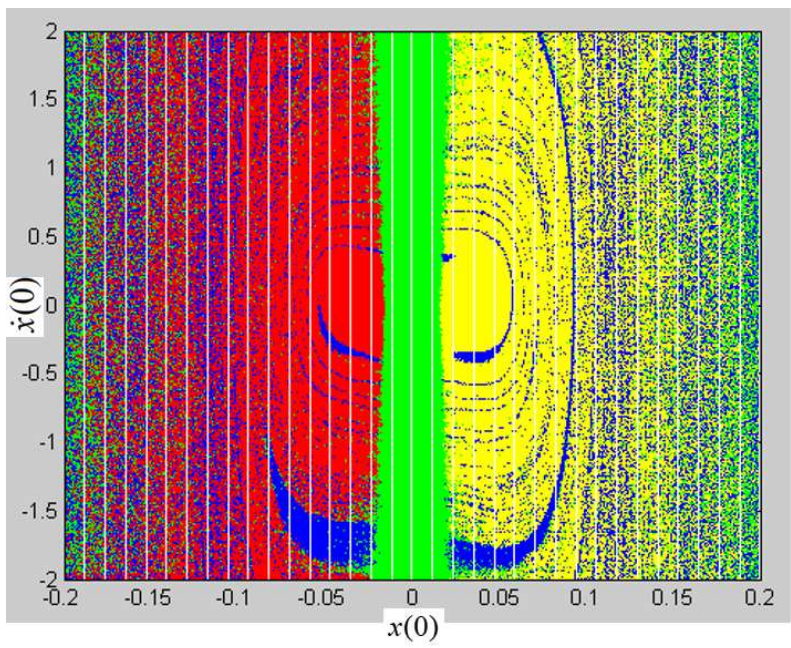

Figure 5. Basins of attraction: the excitation frequency $f=10 \pi$ and the excitation amplitude $A=1.2$. Red, green and yellow colours correspond to basins of intrawell oscillatory solutions around equlibria $1,3,5$, respectively, while blue correspond to basin of interwell oscillations.

corresponding to the excitation frequency and amplitude. To confirm these characteristics, linearly increasing frequency-swept excitation (up-sweep) and linearly decreasing frequency-swept excitation (down-sweep) experiments under the excitation level of acceleration fixed $0.27 \mathrm{~g}$ and $0.35 \mathrm{~g}$ (where $\mathrm{g}$ is the Earth gravitation acceleration) depicted in Fig. 6a and b, respectively. At 0.27 $\mathrm{g}$, the TEH has hysteresis phenomenon (interwell and intrawell orbits) in the frequency range of 7.8-15.9 Hz. At $0.35 \mathrm{~g}$, the corresponding frequency range shifts to 9-17.1 $\mathrm{Hz}$. Therefore, the experiments qualitatively verify the numerical conclusion. (a)

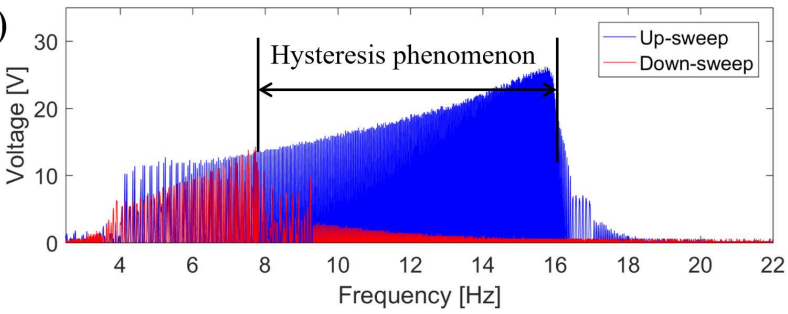

(b)

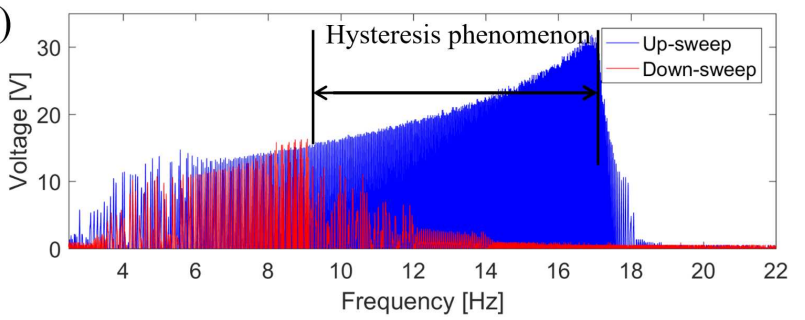

Figure 6. Dynamical hysteresis phenomenon for voltage output of the TEH system in experimental results under linearly increasing frequency sweep excitation (Up-sweep) and decreasing frequency sweep excitation (Down-sweep). (a) and (b) corresponds to different levels of harmonic excitation.

\section{Conclusions}

The main feature of the studied system is the appearance of additional solutions absent in the linear system. This gives the possibilities to adjust various solutions closer to resonance area. Consequently, by changing frequency we observe the specific fitting of the rational frequency response to the frequency of excitation leading to the larger interwell oscillation amplitude of the voltage output. Note that, a chaotic oscillation is also blonging to such a class of interwell solutions. The dynamical hysteresis shown in Fig. 6 is related to the switching between two or more solutions (see Figs. 3-5) during the excitation with variable frequencies. The occurrence of the multiple solutions at a given frequency and also dynamical hysteresis during the frequency sweep give the opportunity to broaden effectively the excitation frequency width of the TEH. This is the most important characteristics in the ambient vibrational energy source where the amplitude and frequency fluctuate.

\section{References}

[1] P.D. Mitcheson, E.M. Yeatman, G.K. Rao, A.S. Holmes, T.C. Green, Proceedings of the IEEE 96, 1457 (2008)

[2] R.L. Harne, K.W. Wang, Smart Mat. Struct. 22, 023001 (2013)

[3] S.P. Pellegrini, N. Tolou, M. Schenk, J.L. Herder, J. Intell. Mater. Syst. Struct. 24, 1303 (2013)

[4] J. Twiefel, H. Westermann, J. Intell. Mater. Syst. Struct. 24, 1291 (2013)

[5] S.P. Beeby, M.J. Tudor, N.M. White, Meas. Sci. Technol. 17, R175 (2006)

[6] S. Zhou, J. Cao, A. Erturk, J. Lin, Appl. Phys. Lett. 102, 173901 (2013) 
[7] A. Syta, G. Litak, M.I. Friswell, S. Adhikari, Eur. Phys. J. B 89, 99 (2016)

[8] T. Huguet, A. Badel, A., M. Lallart, Appl. Phys. Lett. 111, 173905 (2017)

[9] M.I. Friswell, S.F. Ali, S. Adhikari, A.W. Lees, O. Bilgen, G. Litak, J. Intell. Mater. Syst. Struct. 23, 1505 (2012)

[10] A. Erturk, J. Hoffmann, D.J. Inman, Appl. Phys. Lett. 94, 254102 (2009)

[11] F. Cottone, H. Vocca, L. Gammaitoni, Phys. Rev. Lett. 102, 080601 (2009)

[12] S. Stoykov, E. Manoach, G. Litak, Eur. Phys. J. Special Topics 224, 2755 (2015)

[13] M. Borowiec, G. Litak, S. Lenci, Inter. J. Struct. Stab. Dynamics 14, 1440020 (2014)
[14] S. Zhou, J. Cao, D.J. Inman, J. Lin, S. Liu, Z. Wang. Appl. Energy 133, 33-39 (2014)

[15] S. Zhou, J. Cao, D.J. Inman, J. Lin, D. Li, J. Sound. Vib 373, 223 (2016)

[16] S. Zhou, L. Zuo, Commun. Nonlinear. Sci. Numer. Simulat. 61, 271 (2018)

[17] S. Zhou, J. Cao, G. Litak, J. Lin, TM-Tech. Mess. 85, 521-532 (2018)

[18] J. Aguirre, J.C. Vallejo, M.A.F. Sanjuan, Phys. Rev. E 64, 066208 (2001)

[19] G. Litak, M. Coccolo, M.I. Friswell, S.F. Ali, S. Adhikari, A.W. Lees, O. Bilgen, in "Nonlinear oscillations of an elastic inverted pendulum," 2012 IEEE 4th International Conference on Nonlinear Science and Complexity (NSC), Budapest, 2012, pp. 113-116 (2012) 